alternative sampling material for the study of HPV ecology in the male. We present the results from the first study on HPV detection in EPS, as detected using a reference method used at the WHO HPV LabNet global reference laboratory, a multiplex high-throughput genotyping using the Luminex system.

Methods The EPS samples were from 186 heterosexual men (mean age 32 (range 19-60) years (median sexual life start at 17.6 (9-25) years; 33 (1-500) median life-time sex partners; no concurrent STDs at the time of the study) were collected in a urology outpatient unit in St. Petersburg and tested for the presence of HPV DNA in Stockholm by the Luminex assay.

Results The results are presented in the Abstract LBP-1.07 table 1. HPV prevalence in the study population was $25.3 \%$ for all HPV types with $11.8 \%$ for oncogenic types only. High-risk (HR-) HPV 16 and 66 were the most common types 3.7 and $2.7 \%$ of men, respectively. Interestingly, untypable HR-HPV types were found in $3.2 \%$ of EPS samples. Multiple oncogenic types were found in $22.7 \%$ of all HR-HPV + EPS samples.

Conclusions EPS can be used for HPV studies in men: additional investigation of EPS may result in better understanding the transmission of HPV infection and to develop strategies for HPV prevention. For the first time, the full range of HPV types in the EPS of men with no STDs was explored.

Abstract LBP-1.07 Table 1 HPV infection prevalence in the prostate (EPS) samples of 186 men

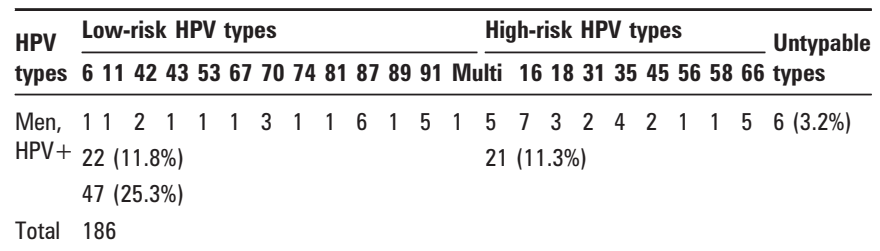

\section{LBP-1.08 PRELIMINARY REPORT ON EXPERIENCE WITH POINT OF CARE SYPHILIS AND HIV TESTING IN HARD-TO-REACH POPULATIONS IN OUTREACH SETTINGS IN EDMONTON, CANADA}

doi:10.1136/sextrans-2011-050119.14

\author{
${ }^{1} \mathrm{~J}$ Bergman, ${ }^{2} \mathrm{~S}$ Plitt, ${ }^{1} \mathrm{~J}$ Gratrix, ${ }^{1} \mathrm{P}$ Conroy, ${ }^{1} \mathrm{~J}$ Li, ${ }^{1} \mathrm{~K}$ Rocco, ${ }^{3} \mathrm{~J}$ Fenton, ${ }^{2} \mathrm{C}$ Archibald, \\ ${ }^{2} T$ Wong, ${ }^{1}$ A Singh. ${ }^{1}$ Alberta Health Services, Edmonton, Canada; ${ }^{2}$ Public Health \\ Agency of Canada, Ottawa, Canada; ${ }^{3}$ Provincial Laboratory for Public Health, \\ Edmonton, Canada
}

Background Edmonton is in the midst of a syphilis outbreak occurring largely in high risk and hard-to-reach populations. Point of care (POC) testing for syphilis and HIV offers the opportunity for immediate and rapid access to testing, and in the case of syphilis, immediate treatment. POC syphilis tests have not been previously evaluated in clinical settings in Canada.

Methods Since 14 February 2011, treponemal syphilis (Bioline Syphilis 3.0) and HIV (INSTI HIV-1/HIV-2 Antibody Test) POC testing, using whole blood from a finger prick specimen, has been offered to outreach clients in Edmonton. POC results were compared to standard testing from simultaneously collected serum specimens. Baseline demographics, sexual and drug use risk behaviour information were collected. Age and gender were collected on individuals who refused POC testing. A descriptive analysis was performed on the characteristics and outcomes of participants and those who refused.

Results As of 15 April 2011, 146 individuals had been offered POC testing; $85.6 \%(n=125)$ consented. Among participants, 59.2\% (74) were male [vs $66.7 \%$ (14) of non-participants, $\mathrm{p}=0.5)$ ] and the median age was 29 yrs (IOR 24-36 yrs) [vs non-participants median age $29 \mathrm{yrs}$ (IOR $25-45 \mathrm{yrs}$ ), $\mathrm{p}=0.2)$ ]. The majority of participants $(83.2 \%$; 104) were heterosexual, $59.2 \%$ (69) were Aboriginal, and $30.4 \%$ (38) reported injection drug use. Among females, $62.7 \%$ (32) reported sex trade involvement, while among males, $21.6 \%$ (16) reported sex with a sex trade worker. Of 121 syphilis treponemal POC tests, 5 (4.1\%) were positive, all in old treated cases of syphilis that were asymptomatic for infectious syphilis at the time of testing. Two syphilis POC $(1.7 \%)$ tests were falsely negative when compared to the standard screening test (Architect Syphilis TP Microparticles, Abbott Laboratories, Illinois, USA); both were in individuals previously treated for syphilis. Of 123 HIV POC tests, 2 (1.6\%) were reactive, both newly diagnosed cases as confirmed by GS HIV-1 Western Blot (BioRad Laboratories, California, USA); both were negative by syphilis POC tests.

Conclusion Preliminary results from Edmonton suggest that POC testing for syphilis and HIV is well accepted among high-risk populations in outreach settings in Edmonton. This ongoing study will assess the utility of these tests in mitigating the further spread of both syphilis and HIV through POC testing, and in the case of syphilis, POC treatment.

\section{LBP-1.09 SERVICES UTILISATION AND HEALTH RELATED VARIABLES OF PERSON LIVING WITH HIV/AIDS IN COMMUNITY IN TAIWAN}

doi:10.1136/sextrans-2011-050119.115

${ }^{1} \mathrm{P}$ Y Chiou, ${ }^{2} \mathrm{~L} \mathrm{C}$ Lin. ${ }^{1}$ Mackay Medicine, Nursing and Management College, Taipei City, Taiwan, China; ${ }^{2}$ Yang-Ming University, Taipei City, Taiwan, China

Background Non-profit organisation (NPO) is a major type to provide the services to response the needs for people living with HIV and AIDS (PLWHA) in the community in Taiwan. The purposes of the study were to describe the services and investigate the effect of the services utilisation of the services on health related variables for PLWHA.

Methods Stratified random sampling and cross-sectional survey methods were used. The self-administered questionnaires include the Demographic, Utilisation and Satisfaction of the Non-Profit Organisation Services Questionnaire, CD4 count and virus load, Customised Adherence Self-Report Questionnaire, Beck Depression Inventory-II (BDI-II), and Medical Outcomes Study Short-Form Health Survey (SF-36) were used to collect date. In Utilisation and Satisfaction of the Non-Profit Organisation Services Questionnaire, the nine services form NPOs were generalised. Data were analysed using forward stepwise multiple regressions.

Results There were 202 study subjects ( $n=178,88.1 \%$ male) and the mean age was 42.54 (SD 10.44) years. The mean virus load and CD4 count were 11031.07 (SD 67153.41) $\mathrm{m} / 1$ and 425.03 (SD 209.30) $\mathrm{mm}^{3}$. The total number of service types which had had accepted by participants was 4.65 (SD 1.67). The services with highest number of users were group or activities $(n=174,86.1 \%)$ and counselling ( $n=137,67.8 \%$ ). Of the 137 participants who had used the counselling service, 126 (92\%) will keep using counselling service. The result indicated that higher frequency of financial aid and counselling services were predictive to the lower depression level of BDI-II $(\mathrm{F}=17.51, \mathrm{R} 2=0.60, \mathrm{p}=0.001)$. Higher frequency of access for medical care service could predict the higher adherence rate to cART of Customised Adherence Self-Report Questionnaire ( $F=6.85, \mathrm{R} 2=0.41$, $p=0.013)$. Higher frequency of access for medical care service and higher adherence rate for cART were collaboratively the predictors to the higher $\mathrm{CD} 4$ count $(\mathrm{F}=17.24, \mathrm{R} 2=0.55, \mathrm{p}=0.009)$. The service of access for medical care, BDI-II, and CD4 were significant with quality of life for SF-36 $(\mathrm{F}=17.31, \mathrm{R} 2=0.66, \mathrm{p}<0.000)$. 